\title{
American College of Medical Genetics Statement on Diagnostic Testing for Uniparental Disomy
}

Lisa G. Shaffer, $P h D^{1}$, Noelle Agan, $M S^{1}$, James D. Goldberg, $M D^{2}$, David H. Ledbetter, $P h D^{3}$, John W. Longshore, $P h D^{4}$, and Suzanne B. Cassidy, $M D^{5}$

\section{OVERVIEW}

Uniparental disomy represents a departure from the usual situation in which one member of each pair of chromosomes (called homologous chromosomes) is normally inherited from each parent. Thus, for each of the 23 pairs of human chromosomes, one is normally inherited from the father and the other from the mother. Uniparental disomy (UPD) is the abnormal situation in which both members of a chromosome pair are inherited from one parent, and the other parent's chromosome for that pair is missing. ${ }^{1}$ Uniparental disomy for some chromosomes is without consequence, but for a few chromosomes can result in abnormality in the affected individual through parent-of-origin differences in gene expression. Serious conditions, including syndromes affecting growth and development, can be the result. Below is an explanation of the mechanisms and consequences of UPD and recommendations for situations in which testing for UPD is indicated.

\section{Mechanisms causing UPD}

UPD arises usually from the failure of the two members of a chromosome pair to separate properly into two daughter cells during meiosis in the parent's germline (nondisjunction). The resulting abnormal gametes contain either two copies of a chromosome (disomic) or no copy of that chromosome (nullisomic), instead of the normal single copy of each chromosome (haploid). This leads to a conception with either three copies of one chromosome (trisomy) or a single copy of a chromosome (monosomy). If a second event occurs by either the loss of one of the extra chromosomes in a trisomy or the duplication of the single chromosome in a monosomy, the karyotypically normal cell may have a growth advantage as compared to the aneuploid cells. UPD results primarily from one of these "rescue" events ${ }^{2}$ (Fig. 1). Other mechanisms can also lead to UPD, including a postfertilization error (via somatic recombination or gene conversion), gametic complementation, and somatic replacement of a derivative chromosome. ${ }^{3,4}$ These mechanisms, with the exception of a postfertilization error,

\footnotetext{
From the ${ }^{1}$ Department of Molecular and Human Genetics, Baylor College of Medicine, Houston, Texas; ${ }^{2}$ Prental Diagnosis Center, California Pacific Medical Center, San Francisco, California; ${ }^{3}$ Department of Human Genetics, University of Chicago, Chicago, Illinois; ${ }^{4}$ Greenwood Genetic Center, Greenwood, South Carolina; and ${ }^{5}$ Department of Pediatrics, Division of Human Genetics, University of California, Irvine, California.

Dr. Cassidy's current address is Department of Pediatrics, Division of Human Genetics, University of California Medical Center, Orange, CA.

Address for reprints: American College of Medical Genetics, 9650 Rockville Pike, Bethesda, MD 20814-3998.
}

will result in UPD of the entire chromosome (holochromosomic). Since the majority of nondisjunction occurs in maternal meiosis I, ${ }^{5}$ it is more likely for a trisomy to consist of two maternal chromosomes and one paternal chromosome. In this case, maternal UPD will result if the paternal chromosome is lost and the two maternal homologues are retained (Fig. 1a). Therefore, in a trisomy rescue, the ensuing UPD will most often result in heterodisomy (inheritance of the two different homologous chromosomes from one parent). Regions of isodisomy (homozygosity of contiguous loci) may result from meiotic recombination. Trisomy associated with Robertsonian translocations may also resolve to disomy through loss of a chromosome and would result in UPD in 50\% of cases (Fig. $2 \mathrm{a})$. The relatively rarer monosomy rescue through duplication of a chromosome during mitosis results in isodisomy for the whole chromosome (two identical homologues). Since most nondisjunction events occur during maternal meiosis I, rescue of a monosomic conceptus through chromosomal duplication (or isochromosome formation, see below) would be expected to result most often in paternal UPD (Figs. $1 \mathrm{~b}$ and $2 b)$.

\section{Situations associated with UPD}

Of the 47 possible types of whole chromosome UPD (maternal and paternal for the 22 autosomes and $\mathrm{X}$, and paternal XY), most have been observed (Fig. 3). Cases of UPD have been identified following (a) the observation of prenatal or postnatal mosaicism (i.e., a mixture of trisomic and normal cells), (b) identification of a structurally abnormal chromosome, (c) molecular investigation of recessive genetic disease, and (d) a phenotype suggestive of a particular syndrome associated with imprinting. Evidence for the trisomy rescue mechanism has come in part from observations of cases of prenatal mosaicism. ${ }^{6,7}$ Discrepancies between karyotypes derived from chorionic villus samples (CVS) and those derived from amniotic fluid specimens have led to the identification of the parental origin of the chromosomes involved in the mosaicism and has uncovered UPD in some cases. ${ }^{2}$ The observation of an abnormal karyotype (aneuploidy) in the placenta at CVS or pathologic exam with a normal fetal karyotype in the amniotic fluid (termed confined placental mosaicism) is now recognized as a risk factor for UPD.

UPD was first identified in humans when a child was found to have cystic fibrosis (CF) due to inheritance of two identical copies of chromosome 7 from only the mother and no contribution from the father. Thus, the child's CF was due to the 


\section{(a) Trisomy Rescue}

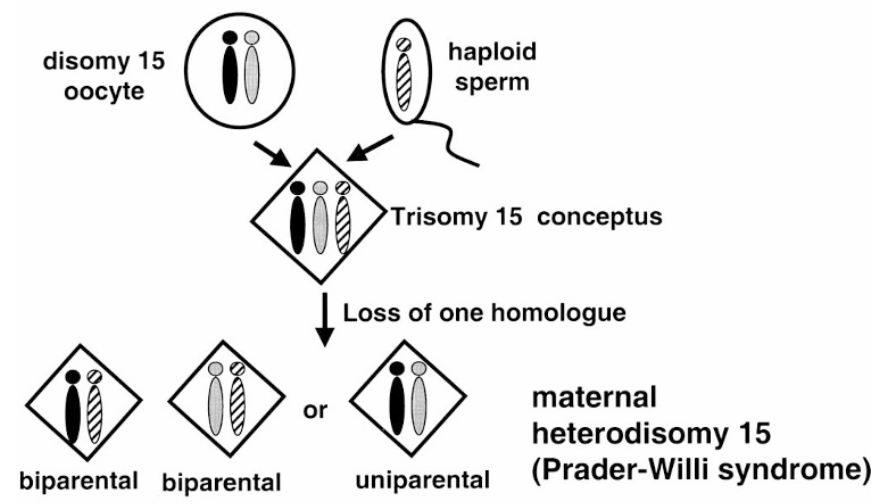

(b) Monosomy Rescue

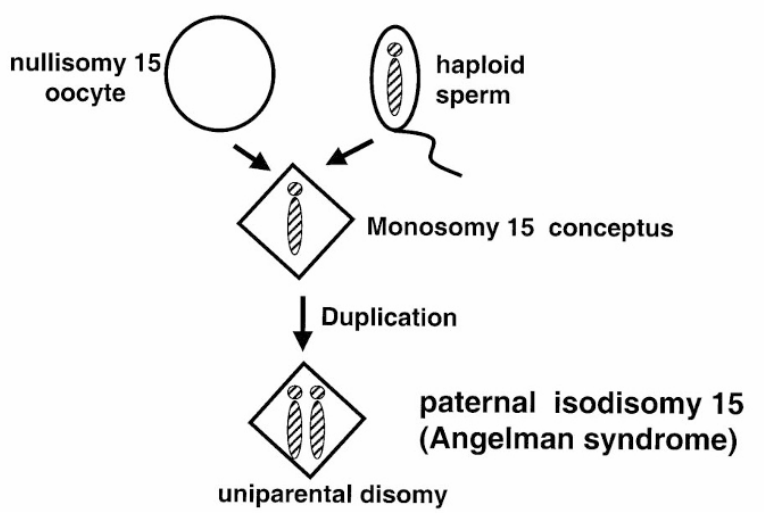

Fig. 1 Common mechanisms resulting in uniparental disomy. (a) Trisomy rescue mechanism. Loss of one homologue in a trisomy conceptus theoretically results in UPD in one-third of cases. The example shown is that of a trisomy 15 conceptus, through which rescue may result in maternal heterodisomy 15 and Prader-Willi syndrome. (b) Monosomy rescue mechanism. Duplication of the only copy of that chromosome in a monosomic conceptus will result in UPD. The example shown is that of a monosomy 15 conceptus in which rescue would result in paternal isodisomy and Angelman syndrome.

presence of two copies of the maternal chromosome 7 that carried the abnormal CF gene as a result of maternal UPD for chromosome $7 .^{3}$ This situation has subsequently been identified for a number of other disorders on several chromosomes. UPD has also been observed to result from the presence of a structurally abnormal chromosome, including Robertsonian translocations, isochromosomes, reciprocal translocations, derivative chromosomes, inversions, and marker chromosomes. Robertsonian translocations and isochromosomes of the acrocentric chromosomes are the most common structural anomalies found in cases of UPD. ${ }^{8}$ This is likely due to the increased risk for aneuploidy in offspring of Robertsonian translocation carriers and the mechanisms associated with isochromosome formation $^{8}$ (Fig. 2).

In many of the UPD cases identified following investigation of single gene disorders by linkage or mutation analyses, the patients did not manifest additional anomalies beyond those expected for their disease.,2,9 However, for a few chromosomes, patients exhibit phenotypic effects due to genomic im- printing. Genomic imprinting is the differential expression of a gene(s) depending on the sex of the transmitting parent. Genomic imprinting is thought to occur for a relatively small number of genes in the human genome (Fig. 3). Thus, some genes (e.g., Prader-Willi syndrome on chromosome 15) are expressed only from the paternally inherited chromosome; whereas other genes (e.g., Angelman syndrome on chromosome 15) are expressed only from the maternally inherited chromosome. Phenotypic abnormalities can result from overexpression of genes in some cases (e.g., Beckwith-Wiedemann syndrome) or lack of gene expression in other cases (e.g., UBE3A and Angelman syndrome). Segments of isodisomy are of additional clinical significance, beyond the possibility of imprinted regions, if they contain a recessive disease allele. ${ }^{10}$

\section{Consequences of UPD}

For the majority of chromosomes, there is no apparent phenotypic effect from UPD (Fig. 3). However, for a few chromosomes, parent-specific imprinting exists and UPD of these chromosomes lead to clinically recognizable consequences (Fig. 3). Only five chromosomes have been shown to have a definite phenotypic effect due to uniparental inheritance of imprinted regions (maternally derived chromosomes 7, 14, and 15; paternally derived chromosomes 6, 11, 14 and 15).2,4,9 For some chromosomes, it is currently unclear if there are phenotypic effects due to imprinting (maternally derived chromosomes 2 and 16 and 20; paternally derived chromosome 20). This uncertainty is due mainly to the subtle nature of the anomalies (e.g., maternal disomy 16), conflicting reports in the literature (e.g., in maternal disomy 2), confounding mosaicism (e.g., maternal disomy 2 and 16), or too few cases reported (e.g., maternal and paternal disomy 20).

\section{Risks for UPD}

The empiric risks for UPD following the observation of prenatal mosaicism for certain chromosomes or a prenatally diagnosed Robertsonian translocation have been recently defined. The chance that trisomy 15 mosaicism, observed prenatally as confined placental mosaicism on analysis of CVS, would result in UPD has been estimated to be $11 \%$ to $25 \% .{ }^{11-13}$ For prenatally identified Robertsonian translocations between nonhomologous chromosomes (e.g., $\operatorname{rob}(13 \mathrm{q} 14 \mathrm{q})$ ), the risk of UPD in the translocation carrier fetus is approximately $0.6 \%{ }^{8}$ For homologous acrocentric rearrangements, for which the majority are isochromosomes (i.e., chromosomes derived from a duplication of a single parental chromosome), the risk of UPD in the balanced carrier fetus is approximately $66 \% .{ }^{8}$

Given these empiric risk figures, clinicians and genetic counselors would benefit greatly from guidelines regarding diagnostic testing and counseling. The following are the recommendations of an American College of Medical Genetics (ACMG) Working Group, which was convened to aid obstetricians, clinical geneticists, cytogeneticists, and genetic counselors in making decisions regarding uniparental disomy diagnostic testing. The document addresses diagnostic testing recommendations for identifying UPD of the entire chromo- 


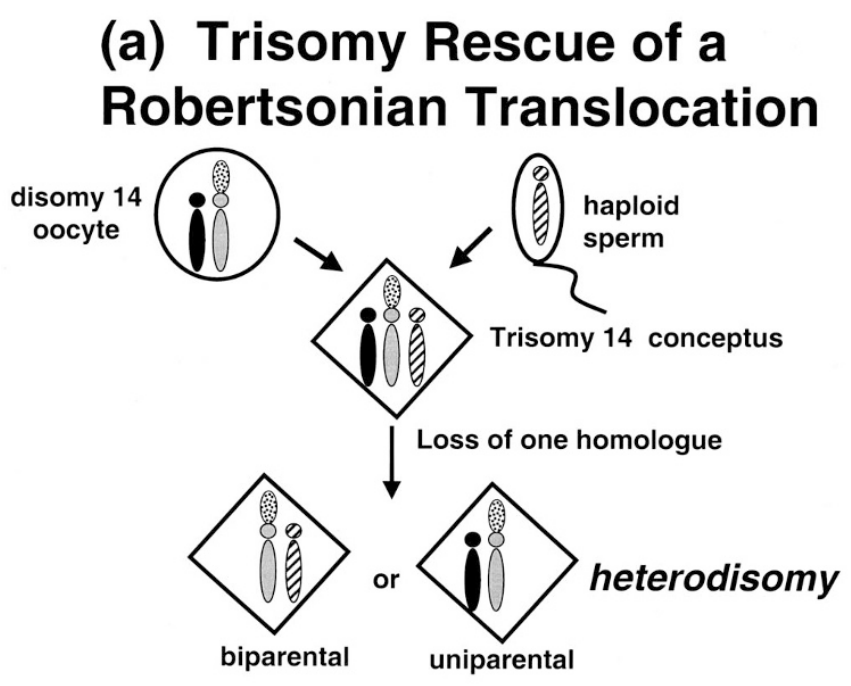

\section{(b) Monosomy Rescue through Isochromosome Formation}

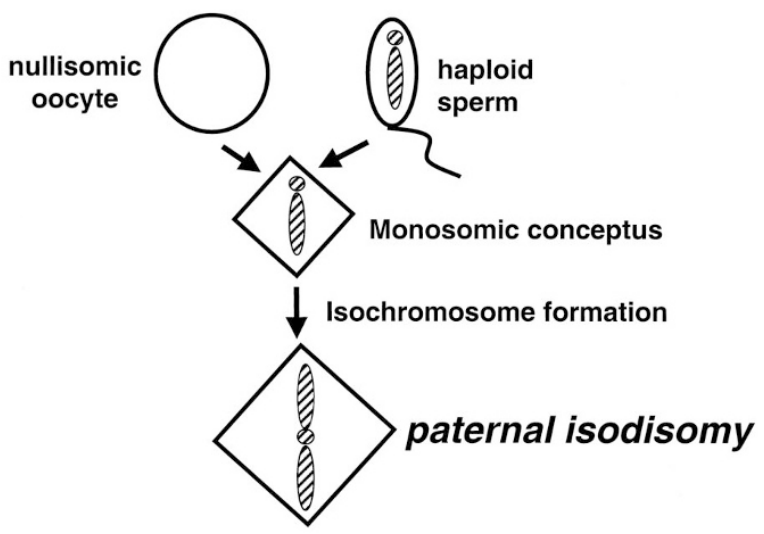

Fig. 2 Common mechanisms resulting in uniparental disomy involving acrocentric chromosomal rearrangements. (a) Trisomy rescue of a trisomy conceptus from a Robertsonian translocation carrier results theoretically in UPD in 50\% of cases. Since the nondisjunction must occur in meiosis I, the resulting UPD would be heterodisomic. (b) Monosomy rescue of a monosomic conceptus resulting from meiosis I nondisjunction and fertilization of a nullisomic gamete. Duplication (through isochromosome formation) of the only copy of a homologue would result in isodisomy in $100 \%$ of cases. Since the majority of nondisjunction occurs in maternal meiosis, most cases of isochromosomes arising through this mechanism would result in paternal isodisomy.

some. Testing recommendations for segmental UPD, mosaic UPD, or UPD restricted to certain tissues, is not addressed specifically in this document.

ASHG/ACMG recommendations for diagnostic testing specific for Prader-Willi and Angelman syndromes have been published previously, ${ }^{14}$ indicating the validity of methylation assays for confirming or excluding the diagnosis of PraderWilli syndrome and Angelman syndrome. Furthermore, the document discussed various approaches to the use of cytogenetic analysis, fluorescence in situ hybridization (FISH) testing, methylation analysis, and UPD testing to distinguish the specific mechanisms involved in these disorders. The current

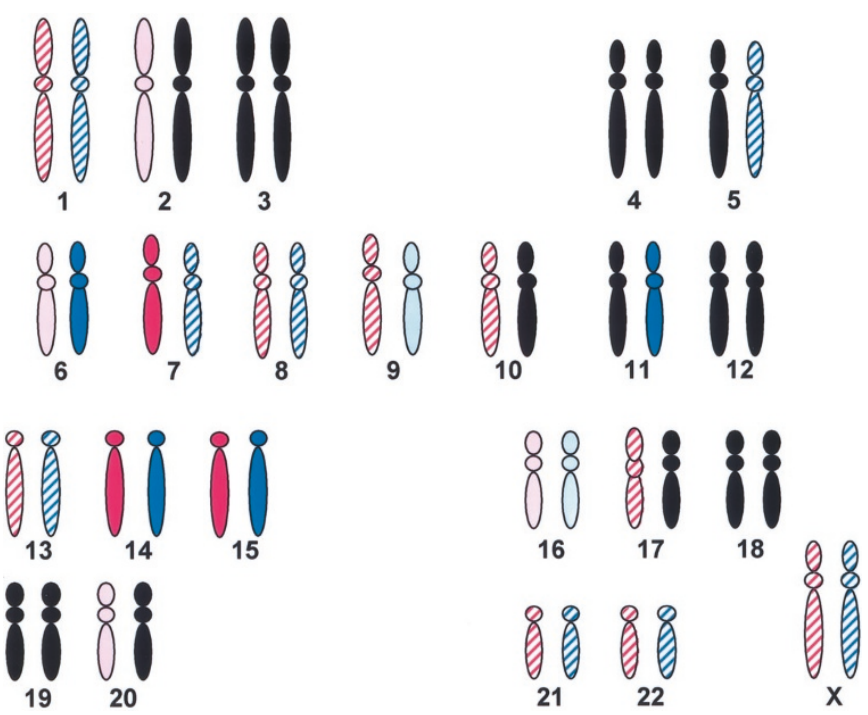

Fig. 3 Schematic representation of UPD cases reported in the literature. Solid black indicates chromosomes in which UPD has not been described. Solid, dark pink or dark blue indicate chromosomes in which maternal or paternal UPD has been described, respectively, and an abnormal phenotype due to imprinting has been established. Solid light pink or light blue indicate chromosomes in which maternal or paternal UPD has been described, respectively, and an abnormal phenotype due to imprinting has not been clearly defined. Hatched pink or blue indicates chromosomes in which maternal or paternal UPD has been described, respectively, and no abnormal phenotype has been identified that could be attributed to imprinting. (Adapted, by permission of Oxford University Press, from Ledbetter and Engel, $1995 .^{2}$ )

document addresses the technical issues for UPD testing of whole chromosomes and can be applied to the situation of Prader-Willi syndrome and Angelman syndrome diagnostic testing.

\section{RECOMMENDATIONS FOR TESTING}

\section{Testing methodology}

DNA-based, polymorphic markers should be utilized when investigating UPD. Short tandem repeat markers (e.g., CArepeats) are used for most UPD studies. These markers are abundant throughout the genome; many have very high heterozygosity values (a reflection of the allele frequency differences in the population) and are ideally suited for multiplex PCR, allowing for 2-4 loci to be tested simultaneously. ${ }^{4,15,16}$ Parental blood samples must be collected and tested along with the fetal or child's sample. The PCR assay can be performed on extracted DNA or cell lysates of uncultured CVS, amniotic fluid, or blood specimens. Multiple markers should be tested for each chromosome of interest. The Committee strongly recommends that at least two fully informative loci, showing either UPD or biparental inheritance, should be obtained for diagnostic reporting. For at least two loci, UPD is evident when a child fails to inherit an allele from one parent for one specific chromosome, but shows biparental inheritance for all other chromosomes investigated. Multiple loci on a single chromosome will distinguish UPD from deletion. For patients showing UPD, at least one other chromosome should be tested to demonstrate biparental inheritance and consistency with cor- 
rect paternity. Since this methodology can potentially uncover nonpaternity, this possibility should be discussed with the family as part of the informed consent for testing. Diagnostic reporting should follow the ISCN 1995 guidelines: uniparental disomy is abbreviated upd (lowercase), followed by the chromosome in parentheses, and then the parental origin [e.g., $\operatorname{upd}(15)$ mat $].{ }^{17}$

There are limitations to this technology in the detection of UPD in samples with somatic mosaicism, segmental UPD, and tissue-specific UPD. The insufficient experience and data in these situations do not allow for specific recommendations for testing at this time.

\section{Chromosomes of clinical relevance}

On any chromosome, two copies of a deleterious single gene for a recessive disorder, through segmental isodisomy, can result in an affected child when only one parent is a carrier. Although this can occur for any recessive allele, currently, it is impossible to identify individuals at risk for this rare event in advance, and these individuals may only be identified if mutation or linkage analyses are being performed or if the individual also has features of a disorder caused by imprinting. Of all possibilities, UPD for only a few chromosomes results in abnormal phenotypes shown to be, or presumed to be, caused by imprinting. These include maternal disomy for chromosomes 7,14 , and 15 and paternal disomy for chromosomes $6,11,14$ and 15.

\section{upd(6)pat and transient neonatal diabetes mellitus}

Transient neonatal diabetes mellitus (DMTN, MIM 601410) is a rare form of diabetes ( 1 in 400,000 to 1 in 500,000 newborns) that usually resolves by 6 months of life. ${ }^{18,19} \mathrm{Al}-$ though the etiology is unclear in the majority of cases, a few cases have been reported to have duplications in the long arm of chromosome $6^{18,20}$ and perhaps about $20 \%$ of cases of DMTN have paternal UPD $6 .{ }^{19}$ The finding of macroglossia or other anomalies in addition to DMTN is a strong indicator to look for UPD. ${ }^{21}$ Recently, a gene involved in cell cycle control, ZAC/PLAGL1, was identified to be imprinted and a good candidate for DMTN. ${ }^{22}$

\section{upd(7)mat and Russell-Silver syndrome}

The syndrome, which is now known as Russell-Silver syndrome (RSS, MIM 180860), was independently described by Silver et al. ${ }^{23}$ and Russell. ${ }^{24}$ RSS is associated with prenatal and postnatal growth retardation with relative sparing of the head, triangular facies and other dysmorphic features, and sometimes limb and facial asymmetry. The molecular etiology for RSS is unclear in the majority of cases. However, about $10 \%$ of patients with RSS have maternal disomy $7 .{ }^{25-28}$ Further evidence for imprinting on chromosome 7 causing RSS comes from the report of two cases of RSS who had duplications in the proximal short arm of chromosome 7. ${ }^{29,30}$ Both duplications included the imprinted gene GRB10, which is thought to be involved in the regulation of growth. Recently, mutations in
GRB10 in two patients with RSS lend support for this gene in the pathogenesis of RSS. ${ }^{31}$

\section{upd(11)pat and Beckwith-Wiedemann syndrome}

Beckwith-Wiedemann syndrome (BWS, MIM 130650) is an overgrowth syndrome associated with macroglossia, organomegaly, omphalocele, and other malformations that has a predisposition to several embryonal tumors, including Wilms tumor. ${ }^{32}$ Neonatal hypoglycemia is common. About $85 \%$ of BWS cases are sporadic, but familial cases have been reported with or without associated chromosomal aberrations involving the most distal band on the short arm of chromosome 11. In chromosomally abnormal cases that produce a partial trisomy for 11p15.5, it has been determined that the origin of the duplicated segment is paternal, when investigated. In contrast, some cases have been reported with apparently balanced translocations or inversions involving $11 \mathrm{p} 15$, and in all cases the abnormality was inherited from the mother. ${ }^{33}$ Partial paternal UPD for the distal short arm of chromosome 11 occurs in about $20 \%$ of BWS patients. ${ }^{34-36}$ Different from the whole chromosome UPD for chromosome 15 that is observed in Prader-Willi syndrome and Angelman syndrome, the UPD in BWS is isodisomic and usually confined to the 11 p15 region. Additionally, mosaicism has been demonstrated at the single cell level. ${ }^{37}$ This indicates that the UPD in BWS is a somatic event rather than a meiotic nondisjunction event. For the remaining patients not demonstrating a chromosome abnormality or UPD, a few demonstrate biallelic expression of the paternally expressed insulin-like growth factor II (IGF2), ${ }^{38}$ a small number of patients have mutations in the cyclin-dependent kinase inhibitor $1 \mathrm{C}\left(p 57^{\mathrm{KIP} 2}\right)$ gene, ${ }^{39}$ and the majority of patients show loss of imprinting for the long QT intronic transcript 1 (LIT1) gene. ${ }^{40}$

\section{upd(14)mat}

Individuals with maternal disomy 14 have short stature, hypotonia, hyperextensible joints, scoliosis, minor facial dysmorphic features, mild developmental delay, and precocious puberty. ${ }^{41}$ Maternal UPD 14 has been reported in association with mosaicism, Robertsonian translocations, and isochromosomes. ${ }^{2,4,9}$ One patient with a partial maternal UPD for chromosomal segments 14q23-14q24.2 who showed developmental delay, mild dysmorphic features, and joint laxity may help to narrow the region in which to search for imprinted genes. ${ }^{42}$

\section{upd(14)pat}

Individuals with paternal disomy 14 have a more severe phenotype than those with maternal disomy 14 , including mental retardation, skeletal abnormalities that result in short-limb dwarfism with narrow thorax, decreased survival due to respiratory difficulties, dysmorphic facies, and scoliosis. ${ }^{41}$ Patients with paternal disomy 14 have been diagnosed following identification of Robertsonian translocations or isochromosomes involving chromosome 14. 


\section{upd(15)mat and Prader-Willi syndrome}

Prader-Willi syndrome (PWS, MIM 176270) is characterized by neonatal hypotonia and poor suck with failure to thrive, developmental delay and/or mental retardation, childhood-onset obesity, short stature, hypogonadism, and severe behavior problems. About 70\% of patients with PWS have a deletion of band $15 \mathrm{q} 12$ on the paternally derived chromosome. About $28 \%$ of cases of PWS result from maternal disomy for chromosome 15 and about $2 \%$ have a defect in the imprinting process. ${ }^{14}$ There are a number of imprinted genes in the PWS critical region, but it is currently not known which of these contribute to the PWS phenotype. ${ }^{43}$

\section{upd(15)pat and Angelman syndrome}

Angelman syndrome (AS, MIM 234400) is associated with severe mental retardation with absent speech, ataxic movements and gait, increased tone after infancy, seizures, and a happy disposition with paroxysmal laughter. About $70 \%$ of patients with AS have a deletion of chromosome band $15 \mathrm{q} 12$ on the maternally inherited chromosome, 3-5\% of cases of AS result from upd(15) pat, ${ }^{14}$ about $5 \%$ of patients have mutations in the ubiquitin-protein ligase E3A (UBE3A) gene, about 3\% have abnormal imprinting by methylation analysis, and the remaining patients $(10-14 \%)$ have no identifiable molecular defect. ${ }^{44}$

\section{Individuals in whom testing should be considered}

- Fetuses with complete trisomy, level II (trisomy in more than one cell in a single primary culture) or level III (trisomy in more than one cell in multiple independent cultures) mosaicism on CVS for chromosomes 6, 7, 11, 14, or 15.

- Fetuses with level II mosaicism on amniotic fluid chromosomes for chromosomes 6, 7, 11, 14, or 15 .

- Fetuses found to carry a Robertsonian translocation or possible isochromosome involving chromosomes 14 or 15. Both familial Robertsonian translocations and de novo translocations are considered to be at risk for UPD.

- Fetuses with anomalies identified by ultrasound that are consistent with features found in UPD syndromes, with or without a structural chromosome abnormality affecting the relevant chromosome.

- Infants or children who present with multiple congenital anomalies, developmental delay or mental retardation, and carry a Robertsonian translocation involving chromosomes 14 or 15 .

- Newborns or infants presenting with neonatal diabetes mellitus.

- Infants or children with features of Russell-Silver syndrome.

- Infants suspected to have Beckwith-Wiedemann syndrome with normal karyotypes and no duplication of 11 p15.5 by fluorescence in situ hybridization (FISH).
- Patients with features of Prader-Willi syndrome or Angelman syndrome who have an abnormal methylation pattern. ${ }^{14}$

\section{SUMMARY OF CLINICAL AND DIAGNOSTIC CONSIDERATIONS}

1. Chromosomes of known clinical relevance include $6,7,11$, 14 , and 15.

2. UPD testing should be considered for

(a) patients presenting with prenatally detected mosaicism or Robertsonian translocations for clinically relevant chromosomes.

(b) patients presenting with features of disorders known to be associated with UPD.

3. Testing should be performed on DNA collected from the mother, father, and child/fetus using polymorphic markers.

4. Reporting of results includes at least two fully informative markers from each chromosome of interest and reported using the ISCN 1995 guidelines. ${ }^{17}$

Approved by the Board of Directors of the American College of Medical Genetics on January 21, 2001.

(C) AMERICAN COLLEGE OF MEDICAL GENETICS, 2001

\section{Acknowledgments}

The authors thank Robert L. Nussbaum (representing the oversight committee), National Institutes of Health, Bethesda, and Jonathan Zonana (representing the oversight committee), Oregon Health Sciences University, Portland, for their guidance; and Bassem Bejjani, Baylor College of Medicine, for his critical review of the manuscript.

\section{References}

1. Engel E. A new genetic concept: uniparental disomy and its potential effect, isodisomy. Am J Med Genet 1980;6:137-143.

2. Ledbetter DH, Engel E. Uniparental disomy in humans: development of an imprinting map and its implications for prenatal diagnosis. Hum Mol Genet 1995;4:17571764 .

3. Spence JE, Perciaccante RG, Greig GM, Willard HF, Ledbetter DH, Hejtmancik JF, Pollack MS, O'Brien WE, Beaudet AL. Uniparental disomy as a mechanism for human genetic disease. Am J Hum Genet 1988;42:217-226.

4. Shaffer LG, McCaskill C, Adkins K, Hassold TJ. Systematic search for uniparental disomy in early fetal losses: the results and a review of the literature. Am J Med Genet 1998;79:366-372.

5. Koehler KE, Hawley RS, Sherman S, Hassold T. Recombination and nondisjunction in humans and flies. Hum Mol Genet 1996;5:1495-1504.

6. Cassidy SB, Lai LW, Erickson RP, Magnuson L, Thomas E, Gendrom R, Herrmann J. Trisomy 15 with loss of the paternal 15 as a cause of Prader-Willi syndrome due to maternal disomy. Am J Hum Genet 1992;51:701-708.

7. Kalousek DK, Langlois S, Barrett I, Yam I, Wilson DR, Howard-Peebles PN, Johnson MP, Giorgiutti E. Uniparental disomy for chromosome 16 in humans. Am J Hum Genet 1993;52:8-16.

8. Berend SA, Horwitz J, McCaskill C, Shaffer LG. Identification of uniparental disomy following prenatal detection of Robertsonian translocations and isochromosomes. Am J Hum Genet 2000;66:1787-1793.

9. Kotzot D. Abnormal phenotypes in uniparental disomy (UPD): fundamental aspects and a critical review with bibliography of UPD other than 15. Am J Med Genet 1999;82:265-274

10. Engel E, DeLozier-Blanchet CD. Uniparental disomy, isodisomy, and imprinting. Probable effects in man and strategies for their detection. Am J Med Genet 1991;40: 432-439. 
11. Robinson WP, Langlois S, Schuffenhauer S, Horsthemke B, Michaelis RC, Christian S, Ledbetter DH, Schinzel A. Cytogenetic and age-dependent risk factors associated with uniparental disomy 15. Prenat Diagn 1996;16:837-844.

12. Christian SL, Smith ACM, Macha M, Black SH, Elder FFB, Johnson JM-P, Resta RG Surti U, Suslak L, Verp MS, Ledbetter DH. Prenatal diagnosis of uniparental disomy 15 following trisomy 15 mosaicism. Prenat Diagn 1996;16:323-332.

13. European Collaborative Research on Mosaicism in CVS (EUCROMIC). Trisomy 15 CPM: probable origins, pregnancy outcome and risk for fetal UPD. Prenat Diagn 1999;19:29-35.

14. Cassidy SB, Beaudet AL, Knoll JHM, Ledbetter DH, Nicholls RD, Schwartz S, Butler MG, Watson M. ASHG/ACMG report. Diagnostic testing for Prader-Willi and Angelman syndromes. Report of the ASHG/ACMG Test and Technology Transfer Committee. Am J Hum Genet 1996;58:1085-1088.

15. Huang TH, Cottingham RW, Ledbetter DH, Zoghbi HY. Genetic mapping of four dinucleotide repeat loci, DXS453, DXS458, DXS454, and DXS424, on the X chromosome using multiplex polymerase chain reaction. Genomics 1992;13:375-380.

16. Shaffer LG, Overhauser J, Jackson L, Ledbetter DH. Genetic syndromes and uniparental disomy: a study of 16 cases of Brachmann-de Lange Syndrome. Am J Med Genet 1993;47:383-386.

17. Mitelman F. ISCN 1995: an international system for human cytogenetic nomenclature. Karger: Basel, Switzerland p. 49.

18. Temple IK, Gardner RJ, Robinson DO, Kibirige MS, Ferguson AW, Baum JD, Barber JC, James RS, Shield JP. Further evidence for an imprinted gene for neonatal diabetes localised to chromosome 6q22-q23. Hum Mol Genet 1996;5:1117-1121.

19. Gardner RJ, Robinson DO, Lamont L, Shield JP, Temple IK. Paternal uniparental disomy of chromosome 6 and transient neonatal diabetes mellitus. Clin Genet 1998; 54:522-525.

20. Arthur EI, Zlotogora J, Lerer I, Dagan J, Marks K, Abeliovich D. Transient neonatal diabetes mellitus in a child with inv dup(6)(q22q23) of paternal origin. Eur J Hum Genet 1997;5:417-419.

21. Christian SL, Rich BH, Loebl C, Israel J, Vasa R, Kittikamron K, Spiro R, Rosenfield $\mathrm{R}$, Ledbetter DH. Significance of genetic testing for paternal uniparental disomy of chromosome 6 in neonatal diabetes mellitus. J Pediatr 1999;134:42-46.

22. Kamiya M, Judson H, Okazaki Y, Kusakabe M, Muramatsu M, Takada S, Takagi N Arima T, Wake N, Kamimura K, Satomura K, Hermann R, Bonthron DT, Hayashizaki Y. The cell cycle control gene ZAC/PLAGL1 is imprinted: a strong candidate gene for transient neonatal diabetes. Hum Mol Genet 2000;9:453-460.

23. Silver HD, Kiyasu W, George J, Deamer WC. Syndrome of congenital hemihypertrophy, shortness of stature, and elevated urinary gonadotrophins. Pediatrics 1953; 12:368-376.

24. Russell A. A syndrome of intrauterine dwarfism recognizable at birth with craniofacial dysostosis, disproportionate short arms and other anomalies. Proc R Soc Med 1954;47:1040-1044

25. Kotzot D, Schmitt S, Bernasconi F, Robinson WP, Lurie IW, Ilyina H, Mehes K, Hamel BCJ, Otten BJ, Hergersberg M, Werder E, Schoenle E, Schinzel A. Uniparental disomy 7 in Silver-Russell syndrome and primordial growth retardation. Hum Mol Genet 1995;4:583-587.

26. Preece MA, Price SM, Davies V, Clough L, Stanier P, Trembath RC, Moore GE. Maternal uniparental disomy 7 in Silver-Russell syndrome. J Med Genet 1997;34:6-9.

27. Eggermann T, Wollmann HA, Kuner R, Eggermann K, Enders H, Kaiser P, Ranke MB. Molecular studies in 37 Silver-Russell syndrome patients: frequency and etiology of uniparental disomy. Hum Genet 1997;100:415-419.
28. Price SM, Stanhope R, Garrett C, Preece MA, Trembath RC. The spectrum of SilverRussell syndrome: a clinical and molecular genetic study and new diagnostic criteria. J Med Genet 1999;36:837-842.

29. Joyce CA, Sharp A, Walker JM, Bullman H, Temple IK. Duplication of 7p12.1-p13, including GRB10 and IGFBP1, in a mother and daughter with features of SilverRussell syndrome. Hum Genet 2000;105:273-280.

30. Monk D, Wakeling EL, Proud V, Hitchins M, Abu-Amero S, Stanier P, Preece MA, Moore GE. Duplication of 7p11.2-p13, including GRB10, in Silver-Russell syndrome. Am J Hum Genet 2000;66:36-46.

31. Yoshihashi H, Maeyama K, Kosaki R, Ogata T, Tsukahara M, Goto Y-I, Hata J-I, Matsuo N, Smith RJ, Kosaki K. Imprinting of human GRB10 and its mutations in two patients with Russell-Silver syndrome. Am J Hum Genet 2000;67:476-482.

32. Weng EY, Mortier GR, Graham JM. Beckwith-Wiedemann syndrome. An update and review for the primary pediatrician. Clin Pediatr 1995;34:317-326.

33. Junien C. Beckwith-Wiedemann syndrome, tumourigenesis and imprinting. Curr Opin Genet Dev 1992;2:431-438.

34. Henry I, Bonaiti-Pellie C, Chehensse V, Beldjord C, Schwartz C, Utermann C, Junien C. Uniparental paternal disomy in a genetic cancer-predisposing syndrome. Nature 1991;351:665-667.

35. Henry I, Puech A, Riesewijk A, Ahnine L, Mannens M, Beldjord C, Bitoun P, Tournada M-F, Landrieu P, Junien C. Somatic mosaicism for partial paternal isodisomy in Wiedemann-Beckwith syndrome: a post-fertilization event. Eur J Hum Genet 1993;1:19-29.

36. Catchpoole D, Lam WW, Valler D, Temple IK, Joyce JA, Reik W, Schofield PN, Maher ER. Epigenetic modification and uniparental inheritance of H19 in Beckwith-Wiedemann syndrome. J Med Genet 1997;34:353-359.

37. Bischoff FZ, Feldman GL, McCaskill C, Subramanian S, Hughes MR, Shaffer LG Single cell analysis demonstrating somatic mosaicism involving $11 \mathrm{p}$ in a patient with paternal isodisomy and Beckwith-Wiedemann syndrome. Hum Mol Genet 1995;4: 395-399.

38. Weksberg R, Shen DR, Fei Y-L, Song QL, Squire J. Disruption of insulin-like growth factor 2 imprinting in Beckwith-Wiedemann syndrome. Nat Genet 1993;5:143-150.

39. Hatada I, Ohashi H, Fukushima Y, Kaneko Y, Inoue M, Komoto Y, Okada A, Ohishi S, Nabetani A, Morisaki H, Nakayama M, Niikawa N, Mukai T. An imprinted gene p5 $7^{\mathrm{KIP} 2}$ is mutated in Beckwith-Wiedemann syndrome. Nat Genet 1996;14:171173.

40. Lee MP, DeBaun MR, Mitsuya K, Galonek HL, Brandenburg S, Oshimura M, Feinberg AP. Loss of imprinting of a paternally expressed transcript, with antisense orientation to KVLQT1, occurs frequently in Beckwith-Wiedemann syndrome and is independent of insulin-like growth factor II imprinting. Proc Natl Acad Sci USA 1999;96:5203-5208

41. Sutton VR, Shaffer LG. A search for imprinted regions on chromosome 14: comparison of maternal and paternal UPD cases with cases of chromosome 14 deletion. Am J Med Genet 2000;93:381-387.

42. Martin RA, Sabol DW, Rogan PK. Maternal uniparental disomy of chromosome 14 confined to an interstitial segment (14q23-14q24.2). J Med Genet 1999;36:633-636.

43. Nicholls RD. Incriminating gene suspects, Prader-Willi style. Nat Genet 1999;23: 132-134.

44. Jiang Y-H, Lev-Lehman E, Bressler J, Tsai T-F, Beaudet AL. Neurogenetics '99 Genetics of Angelman syndrome. Am J Hum Genet 1999;65:1-6.

This statement is designed primarily as an educational resource for medical geneticists and other health care providers to help them provide quality medical genetic services. Adherence to this statement does not necessarily ensure a successful medical outcome. This statement should not be considered inclusive of all proper procedures and tests or exclusive of other procedures and tests that are reasonably directed to obtaining the same results. In determining the propriety of any specific procedure or test, the geneticist should apply his or her own professional judgment to the specific clinical circumstances presented by the individual patient or specimen. It may be prudent, however, to document in the patient's record the rationale for any significant deviation from this statement. 